\title{
Review
}

\section{Acupuncture Treatment for Low Back Pain and Lower Limb Symptoms-The Relation between Acupuncture or Electroacupuncture Stimulation and Sciatic Nerve Blood Flow}

\author{
Motohiro Inoue ${ }^{1}$, Hiroshi Kitakoji ${ }^{1}$, Tadashi Yano $^{2}$, Naoto Ishizaki ${ }^{3}$, Megumi Itoi $^{4}$ and \\ Yasukazu Katsumi ${ }^{4}$
}

${ }^{1}$ Department of Clinical Acupuncture and Moxibustion II, ${ }^{2}$ Department of health Promoting Acupuncture and Moxibustion, ${ }^{3}$ Department of Clinical Acupuncture and Moxibustion I and ${ }^{4}$ Department of Orthopaedic Surgery, Meiji University of Oriental Medicine

To investigate the clinical efficacy of acupuncture treatment for lumbar spinal canal stenosis and herniated lumbar disc and to clarify the mechanisms in an animal experiment that evaluated acupuncture on sciatic nerve blood flow. In the clinical trial, patients with lumbar spinal canal stenosis or herniated lumbar disc were divided into three treatment groups; (i) Ex-B2 (at the disordered level), (ii) electrical acupuncture (EA) on the pudendal nerve and (iii) EA at the nerve root. Primary outcome measurements were pain and dysesthesia [evaluated with a visual analogue scale (VAS)] and continuous walking distance. In the animal study, sciatic nerve blood flow was measured with laser-Doppler flowmetry at, before and during three kinds of stimulation (manual acupuncture on lumber muscle, electrical stimulation on the pudendal nerve and electrical stimulation on the sciatic nerve) in anesthetized rats. For the clinical trial, approximately half of the patients who received Ex-B2 revealed amelioration of the symptoms. EA on the pudendal nerve was effective for the symptoms which had not improved by Ex-B2. Considerable immediate and sustained relief was observed in patients who received EA at the nerve root. For the animal study, increase in sciatic nerve blood flow was observed in $56.9 \%$ of the trial with lumber muscle acupuncture, $100 \%$ with pudendal nerve stimulation and $100 \%$ with sciatic nerve stimulation. Sciatic nerve stimulation sustained the increase longer than pudendal nerve stimulation. One mechanism of action of acupuncture and electrical acupuncture stimulation could be that, in addition to its influence on the pain inhibitory system, it participates in causing a transient change in sciatic nerve blood blow, including circulation to the cauda equine and nerve root.

Keywords: disc herniation - pudendal nerve - sciatic nerve - spinal canal stenosis - spinal nerve root

\section{Introduction}

Clinical acupuncture and moxibustion have frequently been used to treat pain, dysesthesia and other symptoms

For reprints and all correspondence: Motohiro Inoue, Department of Clinical Acupuncture and Moxibustion II Meiji University of Oriental Medicine, Honoda, Hiyoshi-cho, Nantan-shiKyoto 629-0392, Japan.

Tel: ++81-771-72-1181; Fax: ++81-771-72-0326;

E-mail: mo_inoue@meiji-u.ac.jp caused by peripheral nerve disorders such as intractable nerve root disorder. Examples include the treatment of sciatic nerve root pain resulting from herniated lumbar disc, cauda equina intermittent claudication due to spinal canal stenosis and peripheral nerve disorders associated with nerve constriction injury and Type 2 diabetes. Despite the fact that clinical experience has often indicated that acupuncture provides definitive relief for

(C) 2007 The Author(s).

This is an Open Access article distributed under the terms of the Creative Commons Attribution Non-Commercial License (http://creativecommons.org/ licenses/by-nc/2.0/uk/) which permits unrestricted non-commercial use, distribution, and reproduction in any medium, provided the original work is properly cited. 
the treatment of these kinds of peripheral nerve disorders, there have been no reports clarifying either what occurs or its mechanism of action. It has been indicated that the pathology of the above peripheral nerve disorders involves reduced nerve blood flow (1). Cauda equine intermittent claudication is a characteristic symptom of lumbar spinal canal stenosis, while intermittent claudication is caused by reduced blood flow of the cauda equine, nerve root and sciatic nerve due to spinal canal stenosis.

Acupuncture and low frequency electroacupuncture treatment are effective for these symptoms, possibly due to some kind of influence on cauda equine, nerve root and sciatic nerve blood flow. This is a report of the results of clinical trials we conducted focusing on herniated lumbar disc and lumbar spinal canal stenosis, two conditions frequently encountered in the field of clinical acupuncture and moxibustion. With the objective of also investigating the mechanism involved, there is also a commentary concerning results of acupuncture and electroacupuncture stimulation of the sciatic nerve in rats conducted to observe changes in blood flow.

\section{Methods}

\section{Clinical Trial Concerning Spinal Canal Stenosis}

For acupuncture treatment of spinal canal stenosis, (i) acupuncture stimulation of Ex-B2 (paravertebral point) at the lumbar level where the disorder occurs (hereafter 'disorder level Ex-B2') (2), (ii) electroacupuncture stimulation of the pudendal nerve (3) and (iii) electroacupuncture stimulation of the nerve root were conducted $(4,5)$ and the results of each were examined.

\section{Acupuncture Stimulation at Disorder Level Ex-B2}

Subjects. A total of 10 patients (seven males, three females, mean age of $69.3 \pm 7.4$ years) diagnosed with spinal canal stenosis using MRI, CT or X-ray imaging.

Acupuncture method. After determining the lumbar level of the disorder from the symptoms and X-ray and MRI findings, acupuncture of disorder level Ex-B2 (the bilateral area $1-2 \mathrm{~cm}$ outward from the spinous process of the disordered level, at the stiffest point, with an insertion depth of $2 \mathrm{~cm}$ ) was performed for $10 \mathrm{~min}$ (leaving the needle inserted for a set time before removing, needle used: length $40 \mathrm{~mm}$, diameter $0.18 \mathrm{~mm}$ ) one time/week for a total of 10 times.

Assessment. Assessment was conducted 1 week after the completion of 10 stimulations using a numerical scale, taking 10 as the score for the pain and discomfort of low back pain, lower limb pain and lower limb dysesthesia at the time of the first examination. Continuous walking distance was confirmed with reports by the patients themselves at the time of the 10th treatment.

\section{Electroacupuncture Stimulation of the Pudendal Nerve}

Subjects. A total of 11 patients (seven males, four females, mean age of $71.3 \pm 7.6$ ) diagnosed with spinal canal stenosis on orthopedic examination for whom 2 months of general conservative treatment (NSAIDs, hot packs, massage, etc.) was ineffective. The criterion for determining the ineffectiveness of conservative treatment was whether there was an improvement of $\leq 20 \%$ in the patients' low back pain, lower limb pain and lower limb dysesthesia symptoms or continuous walking distance at the time of the first examination. Among the subjects, symptoms of low back pain were observed in 11, lower limb pain in 11, lower limb dysesthesia in 11 and intermittent claudication in seven.

Electroacupuncture method. The point for acupuncture stimulation of the pudendal nerve is located in the gluteal region (at a point, $50-60 \%$ of the distance along a straight line from the posterior superior iliac spine to the lower inner edge of the ischial tuberosity). An acupuncture needle was inserted in this region (6). When the needle reaches the pudendal nerve, a feeling of stimulation arises in the pudendal area. A second acupuncture needle ( $90 \mathrm{~mm}$ long, $0.24 \mathrm{~mm}$ in diameter) was inserted in the same area and the two needles were used as electrodes for low frequency electroacupuncture treatment (stimulation frequency: $10 \mathrm{~Hz}$, stimulation time: $10 \mathrm{~min}$, stimulation strength: enough for stimulation in the pudendal area to be felt). Stimulation was performed a total of eight times (1/week) (Fig. 1).

Assessment. Assessment was conducted a total of three times, that is before the start of general conservative treatment, after 2 months of conservative treatment and after eight stimulations of the pudendal nerve with electroacupuncture. Assessment of low back pain, lower limb pain and lower limb dysesthesia was performed using a visual analogue scale (hereafter referred to as VAS). The VAS featured a $100 \mathrm{~mm}$ straight line, with the left edge of the line representing no symptoms at all and the right edge the worst symptoms experienced so far. Continuous walking distance was confirmed with reports by the patients themselves.

Statistical analysis. All data are expressed as mean values \pm standard deviation. The paired $t$-test was used for changes in low back pain, lower limb pain, lower limb dysesthesia and continuous walking distance before the start of general conservative treatment, after conservative treatment and after electroacupuncture of the pudendal nerve (Statview 4.5 program, SAS Institute Japan). A $P$-value of $<0.05$ was considered a significant difference. 


\section{Electroacupuncture Stimulation of the Nerve Root}

Subjects. A total of 14 patients (eight males, six females, mean age of $74.3 \pm 10.1)$ diagnosed with spinal canal stenosis for whom 2 months of general conservative treatment (NSAIDs, hot packs, massage, etc.) was ineffective.

Electroacupuncture method. Having ascertained that symptoms, X-ray and MRI findings pointed to nerve root disorder, two acupuncture needles (length: $90 \mathrm{~mm}$, diameter: $0.24 \mathrm{~mm}$ ) were inserted in the part of the nerve root that appears peripherally from the intervertebral foramen (external abdominal side of transverse process) under X-ray fluoroscopy, which is similar to the technique of spinal nerve root block. With at least one of the two needles located in a position close enough to

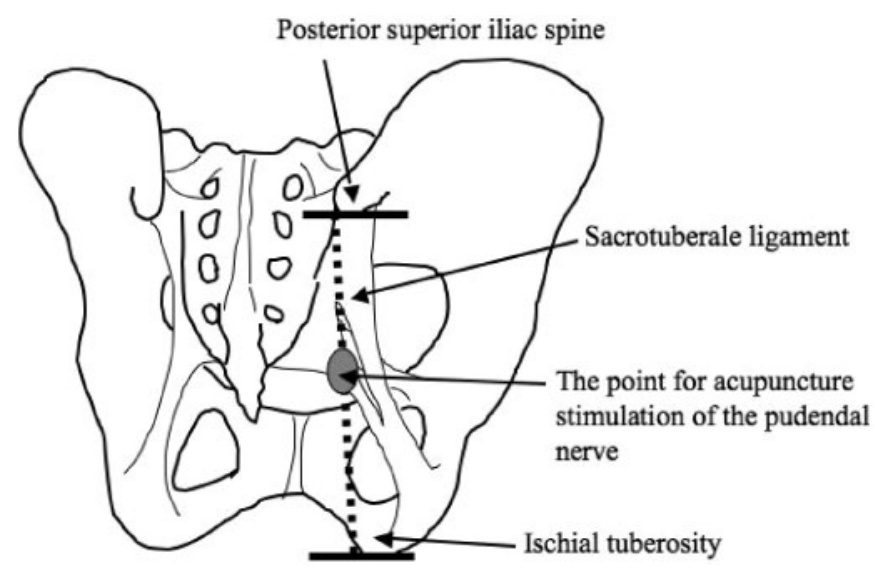

Figure 1. Schematic diagram of electroacupuncture stimulation of the pudendal nerve. The site for electroacupuncture stimulation of the pudendal nerve is located at a point $50-60 \%$ of the distance along a straight line from the posterior superior iliac spine to the lower inner edge of the ischial tuberosity. enable stimulation of the nerve root, electrical acupuncture was conducted using the acupuncture needles as electrodes (stimulation frequency: $2 \mathrm{~Hz}$, stimulation time: $10 \mathrm{~min}$, stimulation strength: degree of stimulation that can be sensed in the area of innervation) (Fig. 2). The treatment session lasted three to five times at 1 week interval.

Assessment. Assessment was conducted before and after each electroacupuncture stimulation of the nerve root (which was performed three to five times depending on the patient) and 3 months after completion of the treatment. Lower limb pain and lower limb dysesthesia were assessed with VAS and continuous walking distance was confirmed with reports by the patients.

Statistical analysis. All data are expressed as mean values \pm standard deviation. After performing one-way analysis of variance for changes in lower limb pain, lower limb dysesthesia and continuous walking distance due to nerve root electroacupuncture stimulation, a multiple comparison test was performed for data obtained before nerve root electroacupuncture stimulation and at various other times using the Bonferroni/Dunn method (Statview 4.5 program, SAS Institute Japan). A $P$-value of $<0.05$ was considered a significant difference.

\section{Clinical Trial Concerning Herniated Lumbar Disc}

For acupuncture treatment of herniated lumbar disc, (i) acupuncture stimulation of Ex-B2 (paravertebral point) at the lumbar level where the disorder occurs (disorder level Ex-B2) (3), (ii) electroacupuncture stimulation of the pudendal nerve (3) and (iii) electroacupuncture stimulation of the nerve root were conducted (3) and the results of each were examined.

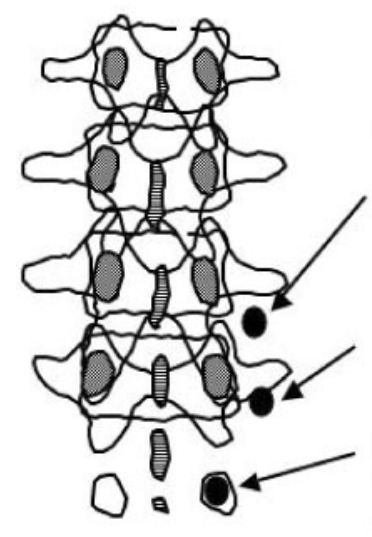

L4 nerve root acupuncture stimulation site

L5 nerve root acupuncture stimulation site S1 nerve root acupuncture stimulation site

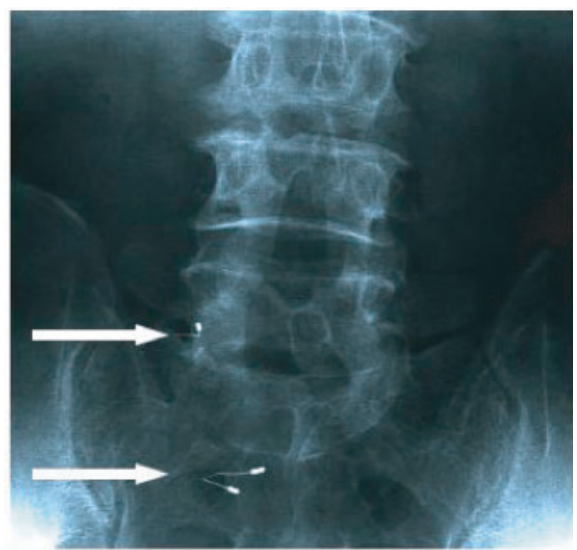

Figure 2. Schematic diagram and X-ray findings of nerve root acupuncture stimulation sits. (Based on the figure Inoue $\mathrm{M}$ et al., Electroacupuncture direct to spinal nerves as an alternative to selective spinal nerve block in patients with radicular sciatica - a cohort study. Acupunct Med 2005; 23: 28). Confirmed the referred pain in the part controlled by the nerve root by inserting two acupuncture needles to the part of the nerve root with disorder under X-ray fluoroscopy. The photograph shows that two acupuncture needles were inserted in the part of the nerve root and connected to the electrode for having electric stimulation. 


\section{Acupuncture Stimulation at Disorder Level Ex-B2}

Subjects. A total of 15 patients (11 males, four females, mean age of $35.3 \pm 5.7$ years) were diagnosed with herniated lumbar disc using MRI, CT or X-ray. Among the subjects, symptoms of low back pain were observed in six, lower limb pain in 12 and lower limb dysesthesia in nine.

Acupuncture method. After determining the lumbar level of the disorder from the symptoms and X-ray and MRI findings, acupuncture of disorder level Ex-B2 (the area $1-2 \mathrm{~cm}$ outward from the spinous process, with an insertion depth of $2 \mathrm{~cm}$ ) was performed for $10 \mathrm{~min}$ (leaving the needle inserted for a set time before removing, needle used: length $40 \mathrm{~mm}$, diameter $0.18 \mathrm{~mm}$ ) one time/week for a total of four times.

Assessment. Assessment was conducted 1 week after the completion of four stimulations using a numerical scale, taking 10 as the score for the pain and discomfort of low back pain, lower limb pain and lower limb dysesthesia at the time of the first examination.

\section{Electroacupuncture Stimulation of the Pudendal Nerve}

Subjects. A total of eight patients (six males, two females, mean age of $35.4 \pm 3.1$ ) were diagnosed as herniated lumbar disc using MRI, CT or X-ray. On orthopedic examination, who did not show amelioration $>50 \%$ with both 3 weeks of general conservative treatment and four acupuncture treatments (one time/ week, $10 \mathrm{~min}$ : leaving the needle inserted for a set time before removing) at Ex-B2 or additional treatment at the points located along with the peripheral route of the disordered nerve were participated in this study. Among the eight subjects, symptoms of low back pain were observed in three, lower limb pain in seven and lower limb dysesthesia in six.

Electroacupuncture method. After determining the lumbar level of the disorder from the symptoms and $\mathrm{X}$-ray and MRI findings, acupuncture was performed at disorder level Ex-B2. Additional treatment at the points located along with the peripheral route of the disordered nerve was carried out depending on the condition of the individual patients (retain the needle at the sites for $10 \mathrm{~min}$ ). These manual acupuncture treatments were combined with the electroacupuncture stimulation of the pudendal nerve.

Electroacupuncture stimulation of the pudendal nerve was performed in the same way as for spinal canal stenosis and for a total of three times (one time/week).

Assessment. Assessment was conducted 1 week after the completion of three stimulations using a numerical scale, taking 10 as the score for pain and discomfort of low back pain, lower limb pain and lower limb dysesthesia at the time of the first examination.

\section{Electroacupuncture Stimulation of the Nerve Root}

Subjects. A total of three patients (two males, one female, mean age of $38.3 \pm 4.3$ years) diagnosed with herniated lumbar disc using MRI, CT or X-ray.

Electroacupuncture method. The same method used for spinal canal stenosis was used to perform nerve root electroacupuncture stimulation.

Assessment. Changes in pain and dysesthesia were assessed before and after each stimulation using a numerical scale, taking 10 as the score for the pain and dysesthesia at the time of the first examination before acupuncture was performed.

\section{Rat Study}

Under the assumption that the effects of acupuncture treatment would be reflected in sciatic nerve blood flow, three kinds of stimulation were conducted, namely (i) acupuncture stimulation of lumbar muscle (7) (8), (ii) electrical stimulation of the pudendal nerve (9) and (iii) electrical stimulation of the sciatic nerve (7) and the effects of each on sciatic nerve blood flow were investigated.

\section{Changes in Sciatic Nerve Blood Flow Due to Acupuncture Stimulation of Lumbar Muscle}

Experimental rats. Thirteen Wistar rats (male, 260-350 g) were used. The rats were administered intraperitoneal doses of urethane $(1.2 \mathrm{~g} / \mathrm{kg})$. Following anesthetization of the rats, rectal temperature was monitored and a body temperature of $37.5 \pm 0.3^{\circ} \mathrm{C}$ maintained with a body temperature control device (MK-900, Muromachi Co. Ltd, Tokyo, Japan). Heart rate was monitored with an electrocardiogram and recorded with a pen recorder (RTA1100, Nihon Kohden Co Ltd, Tokyo, Japan). An incision was made in the necks of the rats, a tracheal cannula inserted and a respirator (SN-4807, Sinano Co Ltd, Tokyo, Japan) connected. A cannula was inserted in the jugular vein for drug administration. A continuous infusion of muscle relaxant $(2 \mathrm{mg} / \mathrm{h})$ was administered with a syringe pump (SCT-525, Terumo Co Ltd, Tokyo, Japan) to immobilize the animals. To measure blood pressure, a cannula containing a solution of heparin and physiologic saline was inserted in the common carotid artery and blood pressure and heart rate were measured continuously with a transducer (P23XL, Sanei Co Ltd, Tokyo, Japan) and recorded with a pen recorder. After this, unilateral exposure of the sciatic nerve was performed, a probe (Type N, Advance Co Ltd, Tokyo, Japan) of the laser-Doppler flowmeter (ALF2100, Advance Co Ltd, Tokyo, Japan) was placed in contact with the exposed nerve and a fixed contact pressure was maintained with a pressure control device (ALF-B, Advance Co Ltd, Tokyo, Japan). Blood flow measured with a time constant of $3 \mathrm{sec}$ 
was recorded with a pen recorder. Measurements were conducted in a paraffin oil pool to prevent drying of the sciatic nerve and surrounding tissue.

Acupuncture stimulation. Manual acupuncture stimulation (the needle was manually rotated after perpendicular insertion to the depth of $10 \mathrm{~mm}$ ) was performed with acupuncture needles (diameter: $0.18 \mathrm{~mm}$, length: $40 \mathrm{~mm}$, Seirin, Japan) in the neighboring muscle of lumbar vertebra L6 (at $10 \mathrm{~mm}$ out from the L6 vertebra spinous process on the side of the rats where blood flow measurements were conducted). The rats were kept in a resting condition for at least $10 \mathrm{~min}$ before acupuncture stimulation, after nerve blood flow, blood pressure and heart rate had become stabilized sufficiently. Rotation stimulation was conducted in the same direction after insertion until the needle stopped and then the needle was left in place for $3 \mathrm{~min}$ before being removed. Each rat received stimulation four to eight times for a total of 58 stimulations.

Statistical analysis. For nerve blood flow data of acupuncture stimulation, values measured every $30 \mathrm{~s}$ before and during stimulation were used, the standard deviation of the measured value before stimulation was calculated for each stimulation and changes during stimulation of double that value (2SD) were taken as indicating an increase or decrease, while values $<2 \mathrm{SD}$ were taken as indicating no change. The same method was used to define increased, decreased or no change in blood pressure and heart rate.

\section{Changes in Sciatic Nerve Blood Flow Due to Efferent Electrical Stimulation of the Pudendal Nerve}

Four Wistar rats (male, 290-310g) were used. Blood pressure, heart rate and sciatic nerve blood flow were measured in the same as was for lumbar electroacupuncture stimulation. Electrical stimulation of the pudendal nerve was performed on the same side as that used to measure sciatic nerve blood flow. In the vicinity of the area used for measurement (gluteal region), the pudendal nerve was exposed and after amputation the distal stump was placed on a platinum bipolar electrode and efferent electrical stimulation $(10 \mathrm{~Hz}, 10 \mathrm{~V}$ and $40 \mathrm{~S})$ was conducted. With the objective of also investigating the mechanism of the response, a muscarinic receptor blocking agent (atropine sulfate) was administered $(1 \mathrm{mg} / \mathrm{kg}$, i.v.) and changes in the response to electrical stimulation were observed.

\section{Efferent Electrical Stimulation of the Sciatic Nerve Changed Sciatic Nerve Blood Flow}

Five Wistar rats (male, 280-340 g) were used. Blood pressure, heart rate and sciatic nerve blood flow were measured in the same as was for lumbar electroacupuncture stimulation. Electrical stimulation of the sciatic nerve was performed on the same side as that used to measure sciatic nerve blood flow. In the vicinity of the area used for measurement (gluteal region), the sciatic nerve was exposed and after amputation the distal stump was placed on a platinum bipolar electrode and efferent electrical stimulation $(10 \mathrm{~Hz}, 10 \mathrm{~V}$ and $30 \mathrm{~S})$ was conducted. With the objective of also investigating the mechanism of the response, a muscarinic receptor blocking agent (atropine sulfate) was administered $(1 \mathrm{mg} / \mathrm{kg}$, i.v.) and changes in the response to electrical stimulation were observed.

\section{Results}

\section{Clinical Trial Concerning Spinal Canal Stenosis}

\section{Acupuncture Stimulation at Disorder Level Ex-B2 Showed Improvement in 6 Out of 10 Cases}

We considered scores of five points or lower after performing acupuncture 10 times (one time/week) as an indication of improved low back pain, lower limb pain and lower limb dysesthesia symptoms. Improvements were observed in 6 of 10 cases for low back pain, 5 of 10 cases for lower limb pain and 3 of 10 cases for lower limb dysesthesia. An increase in continuous walking distance of $100 \mathrm{~m}$ or more compared with the distance at the first examination was taken as indicating improvement. Improvement was observed in 6 of 10 cases. Among them, one case improved from $100 \mathrm{~m}$ at the time of the first examination to $1 \mathrm{~km}$.

\section{Electroacupuncture Stimulation of the Pudendal Nerve Improved Low Back Pain in Only 2 Out of 11 Cases}

VAS scores for low back pain, lower limb pain and lower limb dysesthesia before general conservative treatment were $53.9 \pm 14.5(\mathrm{~mm}, \quad$ mean $\pm \mathrm{SD}), \quad 65.3 \pm 8.1$ and $66.4 \pm 11.1$. Before general conservative treatment, continuous walking distance was $99.3 \pm 41.2$ (mm, mean $\pm \mathrm{SD}$ ). Changes before (after completion of 2 months of general conservative treatment) and after electroacupuncture stimulation of the pudendal nerve, showed an improvement in the symptoms. The score for low back pain improved from $52.5 \pm 15.1$ $(\mathrm{mm}$, mean $\pm \mathrm{SD})$ to $47.1 \pm 13.8(P<0.05)$, for lower limb pain from $63.3 \pm 7.2$ to $40.6 \pm 12.3(P<0.01)$ and for lower limb dysesthesia from $64.1 \pm 10.9$ to $46.3 \pm 17.1$ $(P<0.01)$, while continuous walking distance improved from $103.5 \pm 40.7 \quad(\mathrm{~m}, \quad$ mean $\pm \mathrm{SD})$ to $250.5 \pm 123.3$ $(P<0.05)$. As a criterion before performing electroacupuncture stimulation of the pudendal nerve, a reduction of symptoms of $20 \%$ or more was taken as indicating an improvement. As a result of electroacupuncture stimulation of the pudendal nerve, improvement was seen in low back pain in 2 of 11 cases, in lower limb pain in 8 of 11 
cases and in lower limb dysesthesia in 6 of 11 cases, while continuous walking distance improved in four of seven cases (Fig. 3).

\section{After 2 Months of Conservative Treatment,}

Electroacupuncture Stimulation of the Nerve Root Improved Symptoms

After 2 months of general conservative treatment (before electroacupuncture stimulation of the nerve root), the scores for lower limb pain, lower limb dysesthesia and continuous walking distance were $81.3 \pm 10.4$ $(\mathrm{mm}, \quad$ mean $\pm \mathrm{SD}), \quad 76.9 \pm 19.6$ and $\quad 64.3 \pm 53.5$ $(\mathrm{m}$, mean $\pm \mathrm{SD})$, respectively. Directly after the first nerve root electroacupuncture stimulation, the scores were seen to improve significantly to $29.6 \pm 17.8$ $(P<0.01)$ for lower limb pain, 33.6 $\pm 20.0(P<0.01)$ for lower limb dysesthesia and $148.1 \pm 102.7(P<0.05)$ for continued walking distance. After completion of three to five stimulations, further improvement was seen with scores of $22.0 \pm 18.3(P<0.01)$ for lower limb pain, $21.1 \pm 12.6(P<0.01)$ for lower limb dysesthesia and $224.3 \pm 101.3(P<0.01)$ for continued walking distance. Three months after completion of the final stimulation, there was continued significant improvement with scores of $28.2 \pm 24.8(P<0.01)$ for lower limb pain, $31.1 \pm 22.8$ $(P<0.01)$ for lower limb dysesthesia and $249.3 \pm 99.5$ $(P<0.01)$ for continuous walking distance (Fig. 4).

\section{Clinical Trial Concerning Herniated Lumbar Disc}

\section{Acupuncture Stimulation at Disorder Level Ex-B2 \\ Improved Half the Cases with Low Back Pain (3)}

We considered scores of 5 points or lower after performing acupuncture four times (one time/week) as
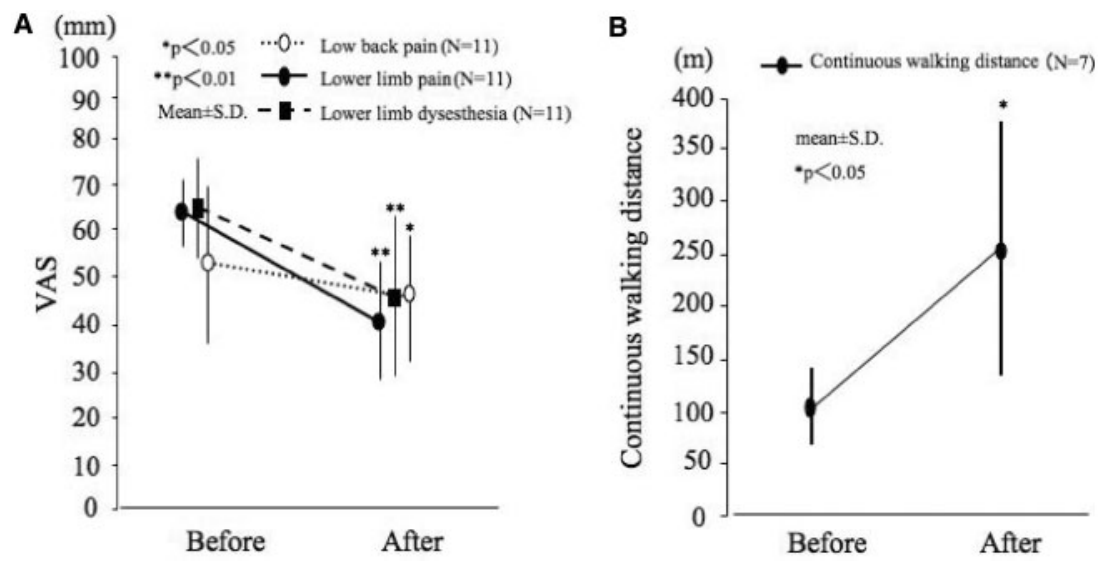

Figure 3. Changes in low back pain, lower limb pain and lower limb dysesthesia due to electroacupuncture stimulation of the pudendal nerve (left). Changes in continuous walking distance due to electroacupuncture stimulation of the pudendal nerve (right).
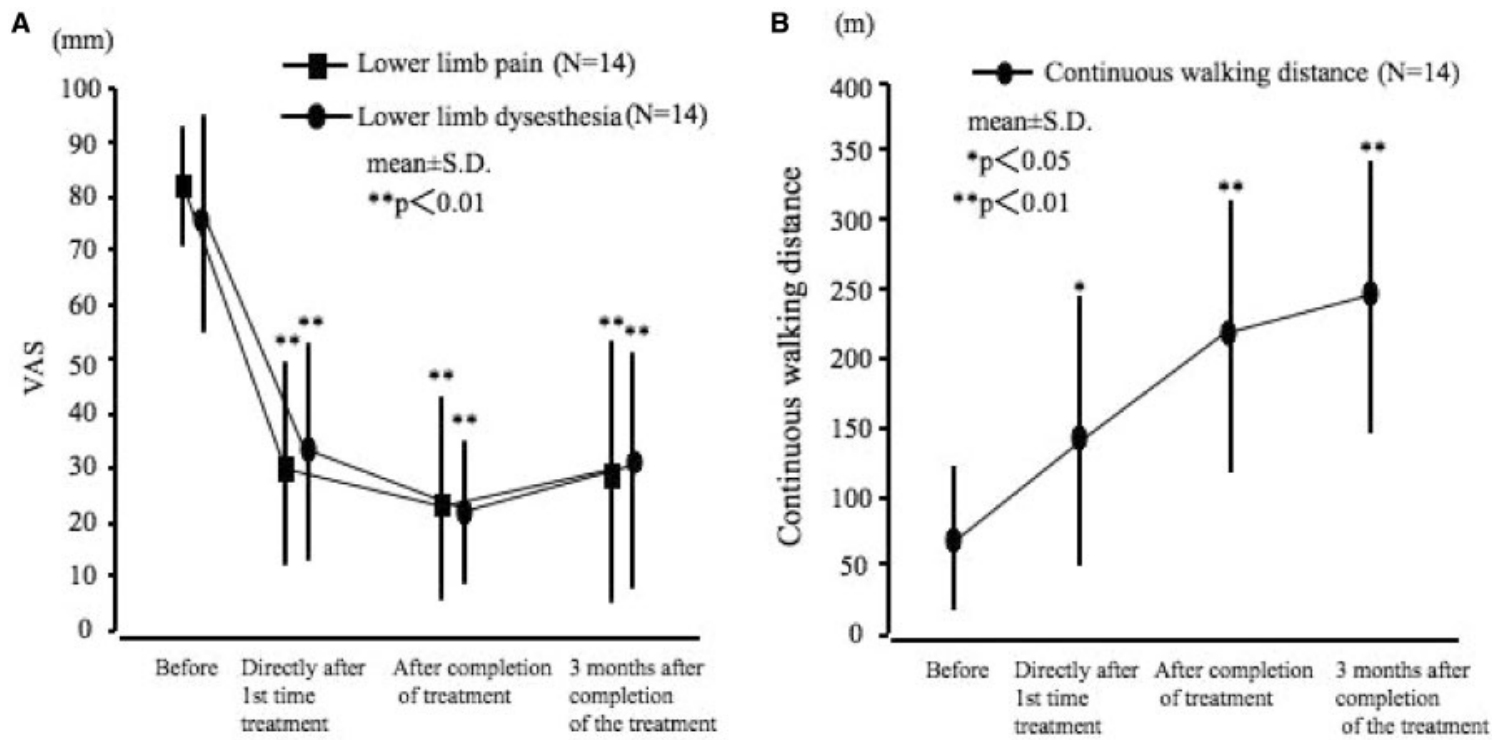

Figure 4. Changes in low back pain, lower limb pain and lower limb dysesthesia due to electroacupuncture stimulation of the nerve root (left). Changes in continuous walking distance due to electroacupuncture stimulation of the nerve root (right). 
an indication of improved low back pain, lower limb pain and lower limb dysesthesia symptoms. Improvements were observed in three of six cases for low back pain, 5 of 12 cases for lower limb pain and three of nine cases for lower limb dysesthesia.

\section{Low Back Pain Improved in Two of Tthree Cases after Electroacupuncture Stimulation of the Pudendal Nerve}

For the eight cases who did not improve more than 50\% after 3 weeks of general conservative treatment followed by four acupuncture treatments at Ex-B2, electroacupuncture stimulation of the pudendal nerve was performed three times (one time/week). As a result, among cases where improvement was below five on the numerical scale, low back pain improved in two of three cases, lower limb pain in three of seven cases and lower limb dysesthesia in three of six cases.

\section{Electroacupuncture Stimulation of the Nerve Root Gave Immediate Relief in All and Long-Term Relief in 2 of 3}

This was performed repeatedly (one time/week) for three cases. The effect directly after the first stimulation was an improvement with the score changing for low back pain from 10 to $2-5$, for lower limb pain from 10 to $1-3$ and for lower limb dysesthesia from 10 to $2-5$. The three cases each received three to five stimulations (one time/ week or 2 weeks) and excluding one case, no clear recurrence in the remaining two cases has so far been observed after 3 and 7 months. In one case, no long-term effect continued and treatment was changed over to surgical treatment.

\section{Rat Study}

\section{Changes in Sciatic Nerve Blood Flow Varied After Acupuncture Stimulation of the Lumbar Muscle}

During acupuncture stimulation to the neighboring muscle of the L6 vertebra, observed reactions ranged from increased and decreased blood flow to no change in blood flow. The results indicated that of the 58 stimulations, with 33 sciatic nerve blood flow increased, with 12 it decreased and with 13 it did not change (Fig. 5). The curves for the increased (upper chart) and decreased (lower chart) cases are indicated in Fig. 6.

Among the 33 stimulations, heart rate increased in eight cases $(24.2 \%)$, decreased in three cases $(9.1 \%)$ and did not change in 22 cases $(66.7 \%)$. Also among the 33 stimulations, blood pressure increased in 15 cases $(45.5 \%)$, decreased in two cases $(6.1 \%)$ and did not change in 16 cases $(48.5 \%)$ (Fig. 7, upper charts). For the stimulations that increased nerve blood, nerve blood flow returned to the value directly before stimulation $9.7 \pm 4.7$ minutes after the discontinuation of stimulation, while

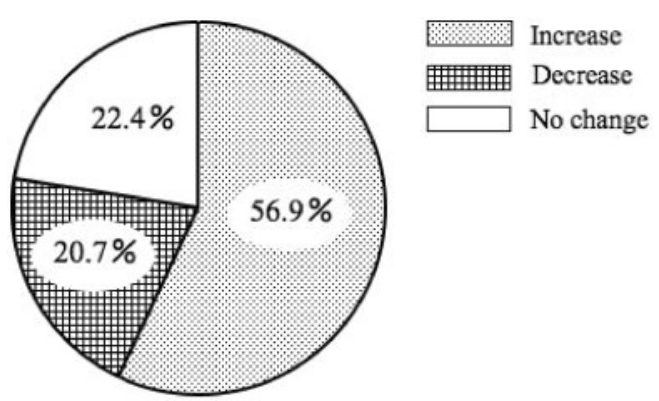

Figure 5. This figure shows the sciatic nerve blood flow responses to 58 acupuncture stimulations to the muscle at L6 level (Ex-B2). (Based on the figure from Inoue $\mathrm{M}$ et al., Effects of lumbar acupuncture stimulation on blood flow to the sciatic nerve trunk. Acupunct Med 2005; 23: 167).
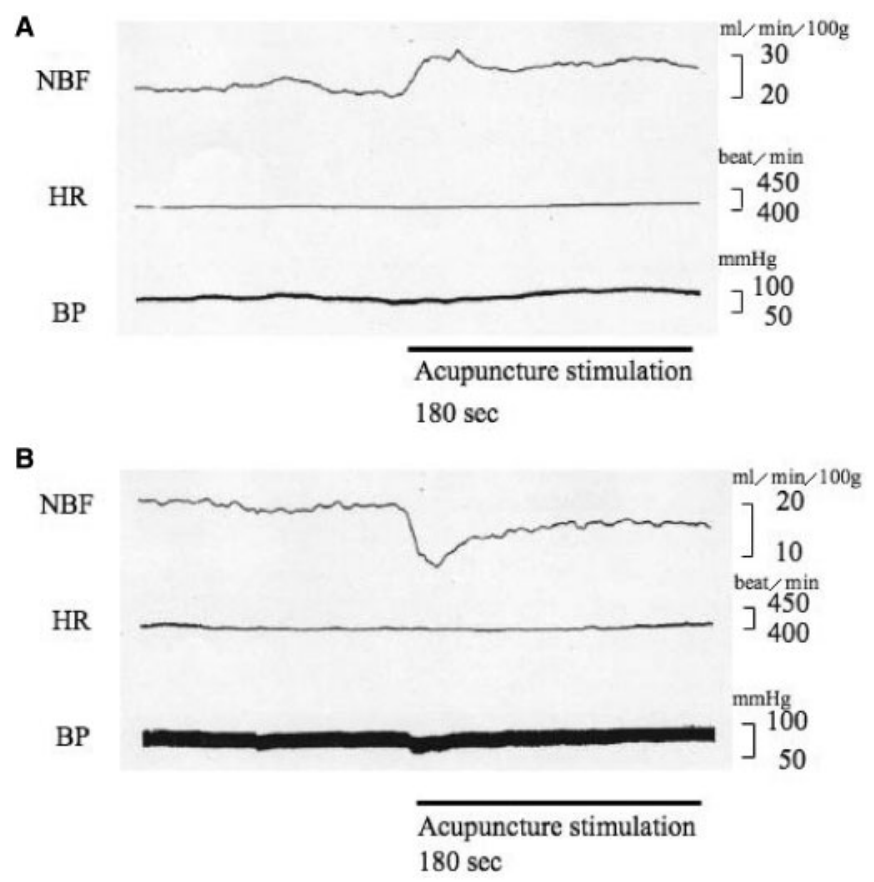

Figure 6. Sciatic nerve blood flow (NBF), heart rate (HR) and blood pressure (BP) changes in a typical case of increased sciatic blood flow (upper chart). Sciatic nerve blood flow (NBF), heart rate (HR) and blood pressure (BP) changes in a typical case of decreased sciatic blood flow (lower chart). (Based on the figure from Inoue $\mathrm{M}$ et al. Effects of lumbar acupuncture stimulation on blood flow to the sciatic nerve trunk. Acupunct Med 2005; 23: 168).

heart rate and blood pressure returned to the pre-stimulus values of $7.2 \pm 6.1$ and $6.8 \pm 3.9$ min after discontinuation of stimulation, respectively.

Among the 12 stimulations, heart rate decreased in three cases $(25 \%)$, increased in two cases $(16.7 \%)$ and did not change in seven cases $(58.3 \%)$. Also among the 12 stimulations, blood pressure decreased in six cases $(50.0 \%)$, increased in two cases $(16.7 \%)$ and did not 


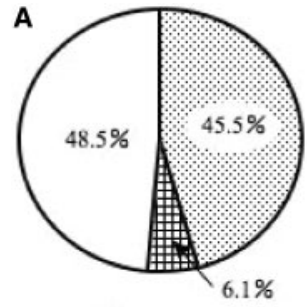

Blood pressure

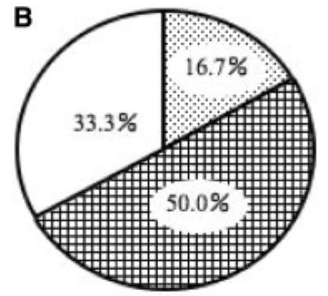

Blood pressure

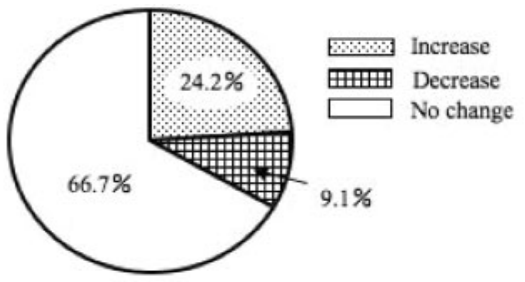

Heart rate

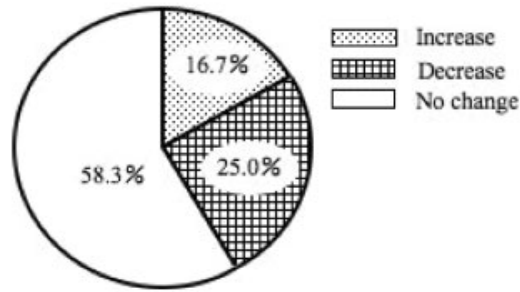

Heart rate

Figure 7. In the 33 cases in which sciatic nerve blood flow increased with acupuncture, this figure shows the proportion of increase, decrease and no change in blood pressure and heart rate (upper charts). In the 12 cases in which sciatic nerve blood flow decreased with acupuncture, this figure shows the proportion of increase, decrease and no change in blood pressure and heart rate (lower charts). (Based on the figure from Inoue $\mathrm{M}$ et al., Effects of lumbar acupuncture stimulation on blood flow to the sciatic nerve trunk. Acupunct Med 2005; 23:168, 169).
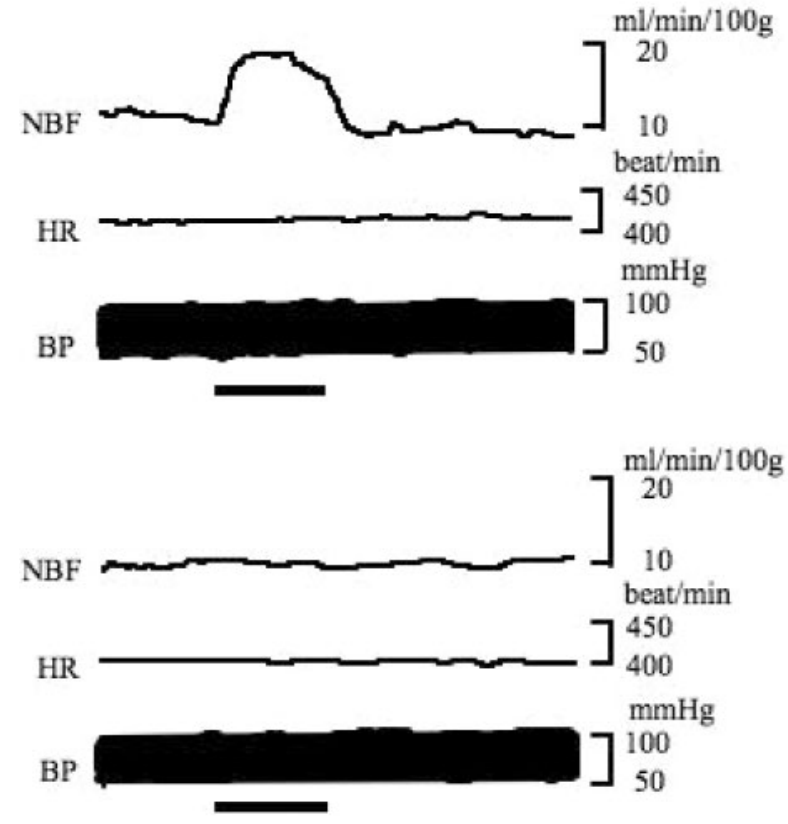

Electrical stimulation $(10 \mathrm{~V}, 10 \mathrm{~Hz}, 40 \mathrm{~s})$

change in four cases $(33.3 \%)$ (Fig. 7, lower charts). For the stimulations that decreased nerve blood, nerve blood flow returned to the value directly before stimulation $7.1 \pm 4.8 \mathrm{~min}$ after the discontinuation of stimulation, while heart rate and blood pressure returned to the prestimulus values of $6.5 \pm 2.5$ and $6.1 \pm 5.4 \mathrm{~min}$ after discontinuation of stimulation.

\section{Sciatic Nerve Blood Flow Increased Immediately With} Efferent Electrical Stimulation of the Pudendal Nerve

Almost identical responses were indicated in three animals, with a typical example shown in Fig. 8. Blood flow to the sciatic nerve started to increase as soon as electrical stimulation of the pudendal nerve began, with a linear increase peaking $10 \mathrm{~s}$ after stimulation began. Thereafter, blood flow tended to decrease gradually during the period of stimulation but increased blood flow was maintained. As soon as stimulation stopped, a linear decrease in blood flow began, returning to almost the same level as that before stimulation $10 \mathrm{~s}$ after stimulation stopped (Fig. 8, upper left).

No clear changes in blood pressure and heart rate due to electrical stimulation were observed. No increased

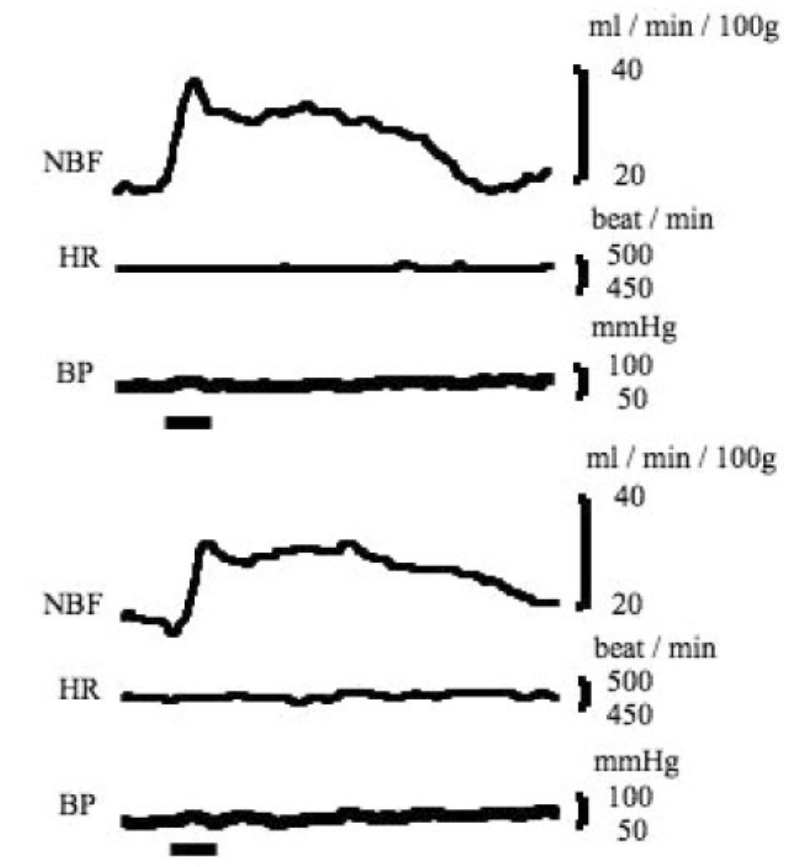

Electrical stimulation $(10 \mathrm{~V}, 10 \mathrm{~Hz}, 30 \mathrm{~s})$

Figure 8. Changes in sciatic nerve blood flow (NBF), blood pressure (BP) and heart rate (HR) due to efferent electrical stimulation of the pudendal nerve and changes in response after administration of atropine (upper left: Before administration of atropine, lower left: After administration of atropine). Changes in sciatic nerve blood flow (NBF), blood pressure (BP) and heart rate (HR) due to efferent electrical stimulation of the sciatic nerve and changes in response after administration of atropine (upper right: Before administration of atropine, lower right: After administration of atropine). 
sciatic nerve blood flow response was observed $5 \mathrm{~min}$ after administration of atropine (Fig. 8, lower left).

\section{Sciatic Nerve Blood Flow Started to Increase as Soon as Electrical Stimulation of the Sciatic Nerve Began}

Almost identical responses were indicated in five animals, with a typical example shown in Fig. 8. Sciatic nerve blood flow started to increase as soon as electrical stimulation began and continued to increase during the period of stimulation, peaking at a rate of $18 \mathrm{ml} / \mathrm{min} /$ $100 \mathrm{~g}$. As soon as stimulation stopped, blood flow gradually decreased, returning to the level before stimulation after about $3 \mathrm{~min}$. No clear changes in blood pressure and heart rate due to electrical stimulation were observed (Fig. 8, upper right). The sciatic nerve blood flow change curve plotted about $5 \mathrm{~min}$ after administration of atropine was almost the same as the pre-dose curve and the peak level was $13 \mathrm{ml} / \mathrm{min} / 100 \mathrm{~g}$, indicating a tendency for a slightly reduced increase after administration of atropine (Fig. 8, lower right).

\section{Discussion}

Acupuncture stimulation at Ex-B2 provided improvement in low back pain and lower limb pain resulting from spinal canal stenosis in $50-60 \%$ of cases. However, a favorable effect on lower limb dysesthesia was observed in only $30 \%$ of patients. An improvement in continuous walking distance was also observed in $60 \%$ of patients, with one patient among them improving from $\sim 100 \mathrm{~m}$ to $1 \mathrm{~km}$.

For patients with lower limb symptoms due to herniated lumbar disc that did not improve after 3 weeks of general conservative treatment, acupuncture stimulation was performed at disorder level Ex-B2. It had a favorable effect on low back pain, lower limb pain and lower limb dysesthesia in $50 \%, 42 \%$ and $33 \%$ of patients. The favorable effect in patients for whom general conservative treatment had been ineffective was particularly significant, indicating the possible effectiveness of acupuncture stimulation at disorder level Ex-B2 as a treatment for nerve root symptoms due to spinal canal stenosis or herniated lumbar disc. Its effectiveness for treating cauda equine intermittent claudication was also indicated and from the viewpoint of the causes of the onset of cauda equine intermittent claudication, it could also cause changes in blood flow to the cauda equine and the sciatic nerve. In the animal study, stimulation increased sciatic blood flow in $33(56.9 \%)$ of 58 stimulations. Among these cases, no relation between increased blood flow and changes in blood pressure were observed in $45.5 \%$. These data indicate that there may be specific factors other than changes in the systemic blood pressure that influence the nerve blood flow by acupuncture stimulation. We suggest that the difference in blood flow response may be due to the state of the subjects being stimulated, or the subtle difference in the stimulated site.

From the above, the mechanism of action of the effect of acupuncture at disorder level Ex-B2 on low back pain and lower limb pain could involve participation by ordinary acupuncture and electroacupuncture stimulation in the activation of the pain inhibitory system (10-12). With regard to improvement of lower limb dysesthesia and continuous walking distance, it was thought that this was possibly due to the effect on sciatic nerve and cauda equine blood flow. Sato et al. (13) reported that electrical stimulation at the dorsal root modified blood flow in the sciatic nerve. Skin and muscle tissue in the vicinity of the vertebrae are supplied by the posterior ramus of the spinal nerves and it is thought that acupuncture stimulation at Ex-B2 causes a change in sciatic nerve and cauda equine blood flow that is a reflex response mediated by these nerves.

Electroacupuncture stimulation of the pudendal nerve was performed on patients with spinal canal stenosis that did not respond to general conservative treatment. Compared with the symptoms before stimulation there was improvement of over $20 \%$, with low back pain, lower limb pain, lower limb dysesthesia and continuous walking distance improving by $18 \%, 73 \%, 55 \%$ and $57 \%$, respectively.

For patients with herniated lumbar disc with $50 \%$ or more of symptoms remaining after 3 weeks of general conservative treatment and acupuncture stimulation of lumbar muscle, electroacupuncture stimulation of the pudendal nerve was also performed. Compared with the symptoms before stimulation there was improvement of over $50 \%$, with low back pain, lower limb pain and lower limb dysesthesia improving by $67 \%, 43 \%$ and $50 \%$, respectively. The effect of electroacupuncture stimulation of the pudendal nerve in patients with symptoms remaining after acupuncture stimulation of lumbar muscle is extremely significant. The results of this trial suggest that it is possibly more effective as a treatment than acupuncture stimulation of lumbar muscle in the vicinity of disorder level Ex-B2 and lower limb muscle. The animal study also indicated that, unlike the acupuncture treatment at Ex-B2, in all cases electrical stimulation of the pudendal nerve increased sciatic nerve blood flow without an accompanying increase in blood pressure.

In light of the above, compared with acupuncture stimulation at Ex-B2, electroacupuncture stimulation of the pudendal nerve could also be a treatment providing a stable, transitory increase in sciatic nerve blood flow. The animal study also indicated that administration of atropine caused the disappearance or decrease of the sciatic nerve blood flow increase response resulting from electrical stimulation of the pudendal nerve. It is highly 
possible that this response is mediated mainly by cholinergic nerves.

For comparatively severe nerve root symptoms that do not respond to general conservative treatment, the treatment at orthopedic departments and pain clinics is nerve root block. Nerve root block is performed by inserting a hypodermic needle as far as the nerve root with the disorder. It could be that its action involves blocking of the transmission route of the pain signal or alleviation of localized inflammation with local anesthetics or steroids $(14,15)$. On the other hand, nerve root electroacupuncture stimulation is a method of treatment that uses nerve block needle insertion procedures to apply low frequency electroacupuncture stimulation to the nerve root with the disorder.

The results of this trial indicate that there is a strong effect directly after nerve root electroacupuncture stimulation that continues for an extended period after a comparatively short period of stimulation. It was also observed in the animal study that efferent electrical stimulation of the sciatic nerve resulted in increased sciatic nerve blood flow over a longer period compared with that provided by electrical stimulation of the pudendal nerve.

In general, the nerve fibers involved in nerve blood flow increase are cholinergic nerves while the axon reflex is related to CGRP (13,16-19). In the animal study this time, the peak value of the sciatic nerve blood flow increase response due to efferent electrical stimulation of the sciatic nerve decreased after administration of atropine. This clearly points to the involvement of vasodilatory nerve fibers in the increase of sciatic nerve blood flow and it is possible that this response is mediated by both cholinergic nerves and the axon reflex.

The biggest difference between nerve root block and nerve root electroacupuncture stimulation is that even though there is stimulation by a needle in both procedures, nerve root block uses a local anesthetic. Consequently, it is thought that when a nerve root block is performed, insertion of the needle provides treatment with stimulation while anesthetic treatment is simultaneously provided by the injection of anesthetic. In other words, nerve root block combines the two reciprocal treatments of anesthesia and stimulation, whereas nerve root electroacupuncture stimulation clearly involves only stimulation treatment.

Further studies are required to clarify the suitability of nerve root block and nerve root electroacupuncture stimulation. Currently, nerve root electroacupuncture stimulation is considered to be unsuitable treatment during the acute phase of severe inflammation. However, it is estimated that it could be effective treatment for chronic nerve root symptoms after the acute phase such as decreased nerve blood flow and delayed axonal flow.

\section{Conclusion}

This is a report of the clinical results of acupuncture stimulation at disorder level Ex-B2, electroacupuncture stimulation of the pudendal nerve and electroacupuncture stimulation of the nerve root for the treatment of spinal canal stenosis and herniated lumbar disc. The results of an animal study of changes in sciatic nerve blood flow due to acupuncture stimulation of lumbar muscle, electric stimulation of the pudendal nerve and electric stimulation of the sciatic nerve are also provided.

The results indicate that acupuncture stimulation at disorder level Ex-B2 provides relief to a certain degree, but for many cases it had no effect and no stable increase in sciatic nerve blood flow was observed. On the other hand, electroacupuncture stimulation of the pudendal nerve was effective even for cases that obtained no effect from stimulation at disorder level Ex-B2. The animal study also indicated a stable sciatic nerve blood flow increase response. With electroacupuncture stimulation of the nerve root the effect was strongest directly after stimulation, and there were cases where a short period of stimulation provided a long-term effect. In the animal study, the blood flow increase response lasted longer than that provided by electroacupuncture stimulation of the pudendal nerve.

From this it can be seen that clinically, the first choice would be acupuncture stimulation at disorder level Ex-B2, which is easy, safe and effective to a certain degree. If it proves to be ineffective, treatment should be changed to electroacupuncture stimulation of the pudendal nerve. The reason why electroacupuncture stimulation of the pudendal nerve is not the first choice is that it is not necessarily easy, and it sometimes causes discomfort in the pudendum.

In cases where electroacupuncture stimulation of the pudendal nerve is also ineffective, treatment should be changed to electroacupuncture stimulation of the nerve root. In addition to being effective, electroacupuncture stimulation of the nerve root uses a needle that is thinner than a nerve block needle and it is extremely safe because no drugs are used. However, the acupuncture techniques required for electroacupuncture stimulation of the nerve root are difficult. Another disadvantage is that strong pain and discomfort can occur in the region of innervation during needle insertion.

It is highly possible that the mechanisms of action of acupuncture stimulation at disorder level Ex-B2, electroacupuncture stimulation of the pudendal nerve and electroacupuncture stimulation of the nerve root involve pain inhibition due to needle stimulation or electrical stimulation (10-12). However, in addition to this, it is thought that repeated transitory changes in blood flow to the sciatic nerve and cauda equine possibly play a role in improving symptoms. 


\section{References}

1. Rydevik B, Brown MD, Lundborg G. Pathoanatomy and pathophysiology of nerve root compression. Spine 1984;9:7-15.

2. Inoue M. Developing a new acupuncture treatment for stenosis in the lumbar part of spinal cord: A basic, clinical review. Japan $J$ Acupunc Manual Therapies 2003;62:40-6 (in Japanese).

3. Yano T, Inoue M. Effect of acupuncture stimulation on blood flow in the nerve trunk. Auton Nerv Syst 2006;43:348-56.

4. Inoue M, Hojo T, Katayama K, Yano T, Katsumi Y. Application of electrical acupuncture at lumbar nerve root as treatment of sciatica due to radiculopathy. Bull Meiji Univ Orient Med 2002;30:1-8 (in Japanese)

5. Inoue M, Hojo T, Yano T, Katsumi Y. Electroacupuncture direct to spinal nerves as an alternative to selective spinal nerve block in patients with radicular sciatica - a cohort study. Acupunct Med 2005;23:27-30.

6. Kitakoji H, Kitamura S, Matsuoka K, Kaneda M, Nakamura T. Anatomical consideration of the acupuncture to the pudendal nerve. J Japan Soc Acupunct Moxibustion 1989;39:221-8, (in Japanese).

7. Inoue $\mathrm{M}$, Katsumi $\mathrm{Y}$, Kawakita $\mathrm{K}$, Okada $\mathrm{K}$, Nakamura $\mathrm{T}$, Matsumoto T. Effects of lumbar acupuncture stimulation and sciatic nerve electrical stimulation on blood flow to the sciatic nerve trunk. J Japan Soc Acupunct Moxibustion 1998;48:130-40, (in Japanese).

8. Inoue M, Hojo $\mathrm{T}$, Yano $\mathrm{T}$, Katsumi $\mathrm{Y}$. Effects of lumbar acupuncture stimulation on blood flow to the sciatic nerve trunk: an exploratory study. Acupunct Med 2005;23:166-70.

9. Inoue M, Hojo T, Ikeuchi M, Katayama K, Ochi H, Katsumi Y. The effect of the electrical acupuncture at pudendal nerve for intermittent claudication of the lumbar spinal canal stenosis. J Japan Soc Acupunct Moxibustion 2000;50:11-9, (in Japanese).

10. Pomeranz B, chiu D. Naloxone blockade of acupuncture analgesia, endorphin implicated. Life Sci 1976;19:1757-62.

11. Sjolund BH, Eriksson MBE. The influence of naloxone on analgesia produced by peripheral conditioning stimulation. Brain Res 1979;173:295-301.

12. Sheng-xing Ma. Neurobiology of Acupuncture: Toward CAM. Evid Based Complement Alternat Med 2004;1:41-7.

13. Sato A, Sato Y, Uchida S. Blood flow in the sciatic nerve is regulated by vasoconstrictive and vasodilative nerve fibers originating from the ventral and dorsal roots of the spinal nerves. Neurosci Res 1994;21:125-33.

14. Macnab I. Negative disc exploration: An analysis of the causes of nerve root involvement in sixty-eight patients. $J$ Bone Joint Surg 1971;53-A:891.

15. Hildebrandt J. Relevance of nerve blocks in treating and diagnosing low back pain is the quality decisive? Schmerz 2001;15:474-83.

16. Zaccheo D, De Michele M, Mancini M, Amenta F. Decreased density of beta-adrenergic and muscarinic cholinergic receptor sites in the vasa nervorum of aged rats. Mech Ageing Dev 1991;60:255-65.

17. Zochodne DW, Ho LT. Influence of perivascular peptides on endoneurial blood flow and microvascular resistance in the sciatic nerve of the rat. $J$ Physiol 1991;444.

18. Zochodne DW, Ho LT. Stimulation-induced peripheral nerve hyperemia: mediation by fibers innervating vasa nervorum. Brain Res 1991;546:113-18.

19. Zochodne DW, Ho LT. Hyperemia of injured peripheral nerve: sensitivity to CGRP antagonism. Brain Res 1992;598:59-66.

Received July 26, 2006; accepted April 12, 2007 


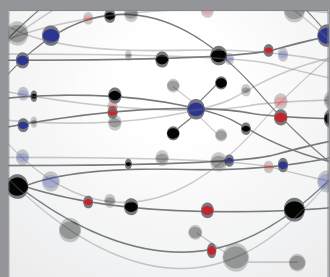

The Scientific World Journal
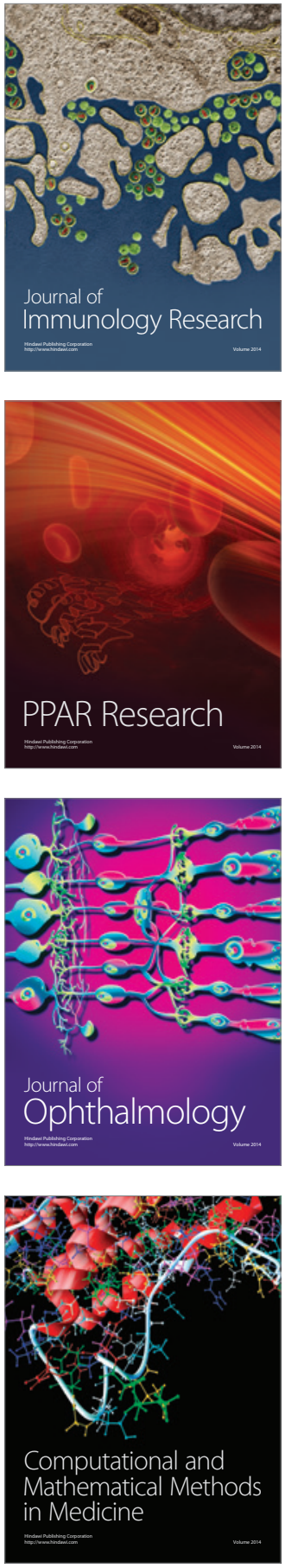

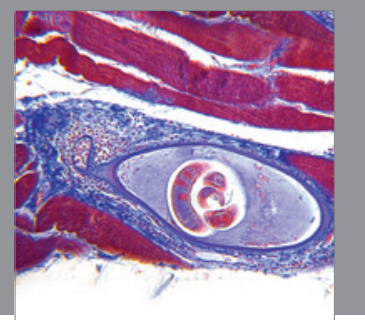

Gastroenterology

Research and Practice
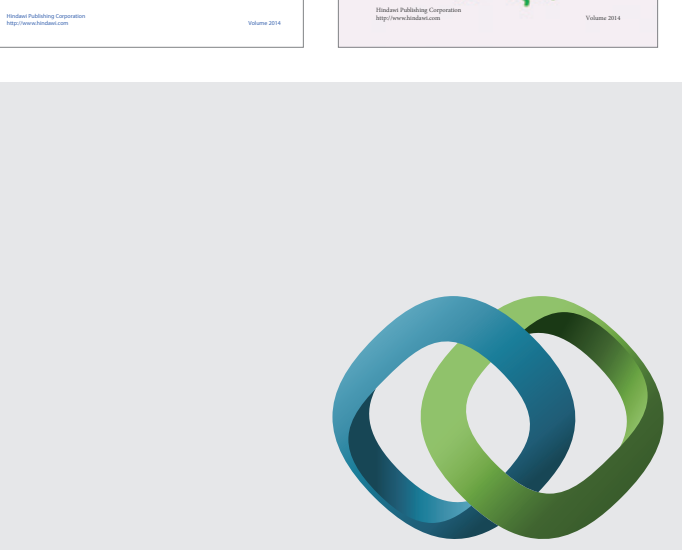

\section{Hindawi}

Submit your manuscripts at

http://www.hindawi.com
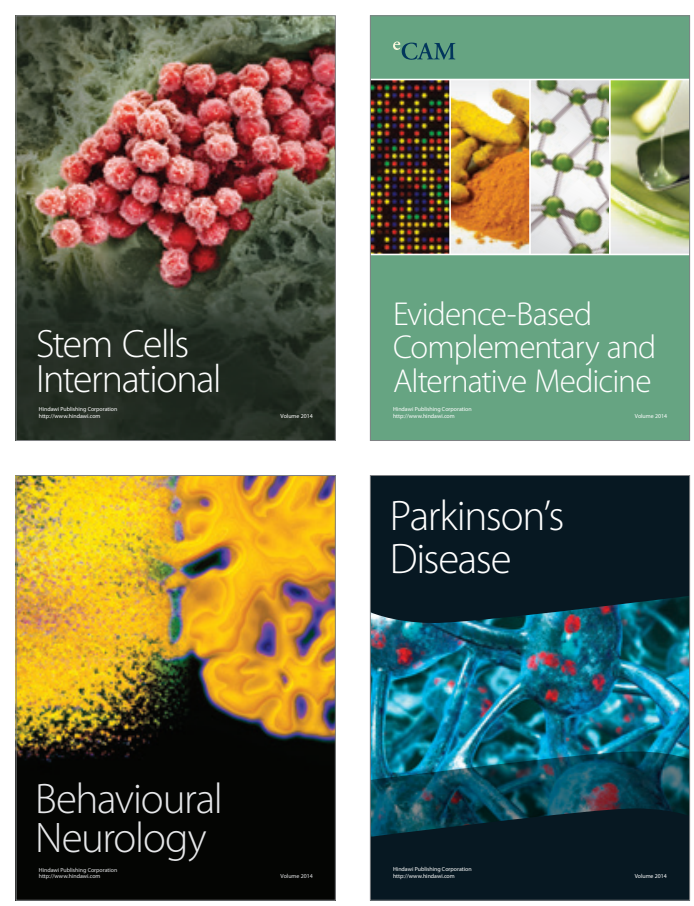

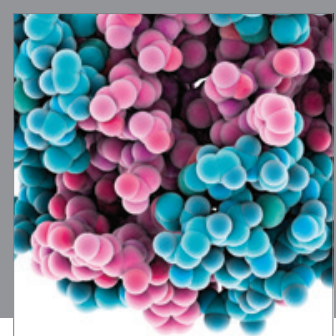

Journal of
Diabetes Research

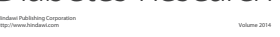

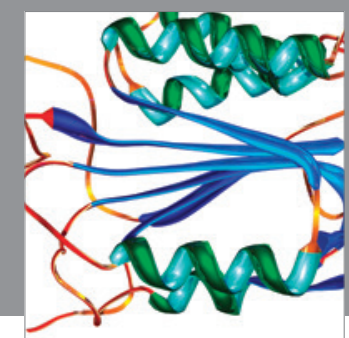

Disease Markers
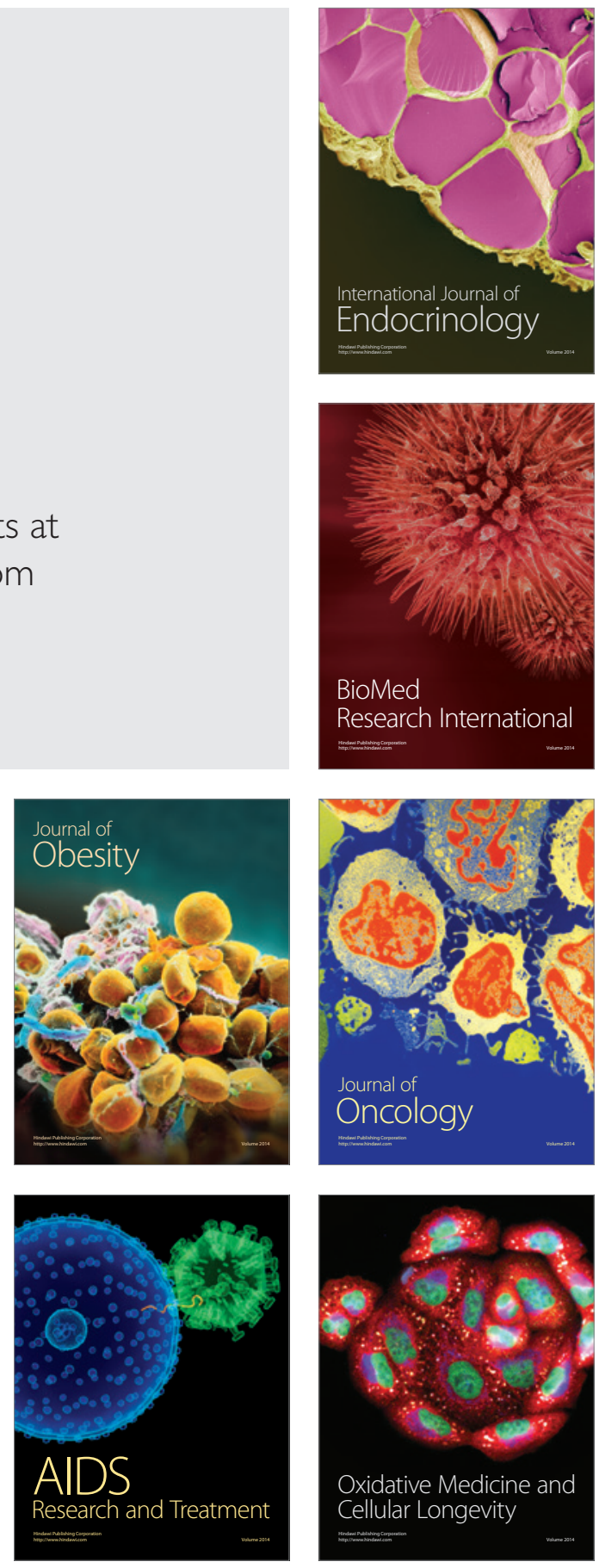